\title{
Mammary-Type Myofibroblastoma of the Elbow: A Case of a Rare Upper Extremity Tumor
}

\author{
RW Ramsey ${ }^{3 *}$, Emily F Mason ${ }^{2}$ and Julia Mayberry ${ }^{1}$ \\ ${ }^{1}$ Department of Hand and Upper Extremity Surgery, USA \\ ${ }^{2}$ Department of Pathology, USA \\ ${ }^{3}$ Orthopedic Surgery Residency, USA \\ *Corresponding author: Randle Ramsey ,4190 City Ave, Philadelphia PA, 19026, USA
}

Submission: 眥 September 22, 2017; Published: 眥 February 27, 2018

\begin{abstract}
Mammary-type myofibroblastoma is a rare spindle cell tumor often found in the breast tissue and trunk of older males and post-menopausal females. The tumor is benign with a sporadic presentation, but some suggest an association with sex steroids. We present a case of a 58-year-old male with a fast growing tumor of the elbow and forearm that was initially suspicious for sarcoma.
\end{abstract}

\section{Introduction}

Mammary-typemyofibroblastoma (MMFB) is a rare tumor often found in the breast tissue of older males as was originally described in 1987 by Wargotz [1]. MMFB belongs to the family of benign stromal cell tumors, which includes solitary fibrous tumors, nodular fasciitis, pseudoangiomatous stromal hyperplasia and primary mammary fibromatosis, all of which are characterized by the presence of myofibroblasticcells $[2,3]$. Classic type MMFB is a bland spindle cell tumor with features of fibroblastic and myofibroblastic differentiation [4].

MMFB most commonly presents as a slow growing, painless mass that develops over the course of months to years $[5,6]$. Though often found in the breast, the tumor also occurs in extramammary sites commonly arising from subcutaneous tissue of the trunk, from the mid-axilla to the groin, which has led to a suspected origin of the embryonic mammary lines. While other locations have been described, MMFB in the limbs is rare [2]. We report a case of histologically confirmed left upper extremity mammary type myofibroblastoma in a 58 year old male with an unusual clinical presentation.

\section{Case Report}

A 58 year old male with a past medical history of hypertension, gout and type II diabetes presented at our institution with a mass in his left elbow that had become apparent over the course of three months. He possibly recognized it six months before the initial office visit, but over the three months prior to presentation, it doubled in size, and became tense and uncomfortable. There was no reported history of trauma and family history was noncontributory.

On physical exam, there was no overlying erythema or ecchymosis. The mass was hard and immobile, measuring approximately $8 \times 6 \mathrm{~cm}$ in greatest diameter. The patient had full active and passive range of motion of the affected extremity and no neurological deficit.

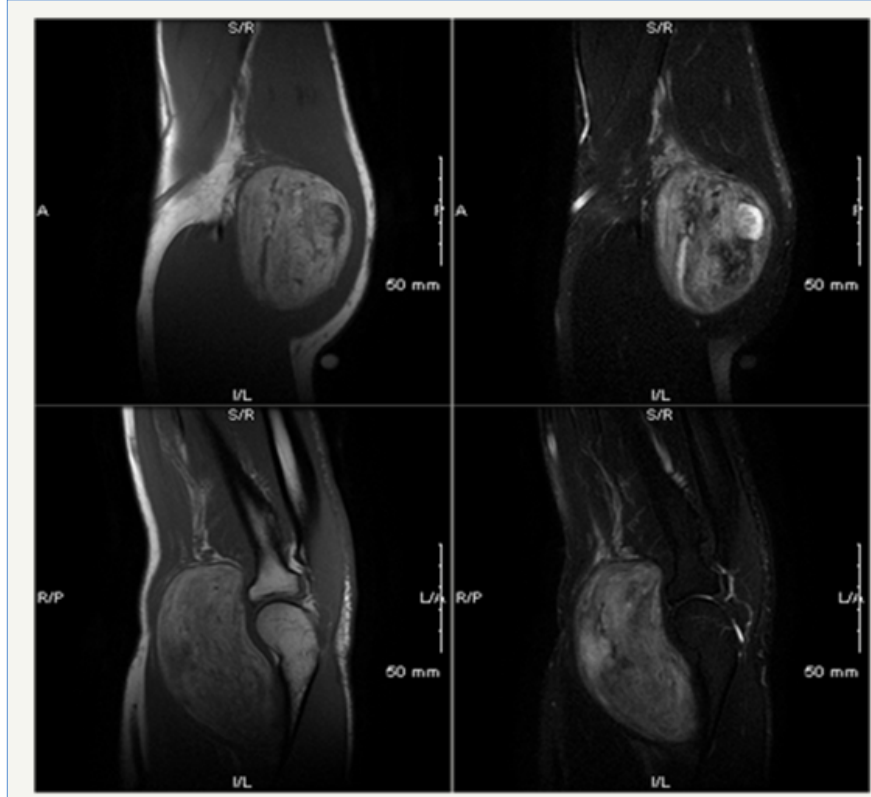

Figure 1: A. Coronal T1 demonstrating a large soft tissue mass, B. Coronal T2 Fat-suppressed post-contrast image demonstrating lesional enhancement, C. Sagittal T1 showing location over anterior radiocapitellar joint, D. T2 fat-suppressed image showing post contrast enhancement.

Plain films taken in the office demonstrated no bony involvement. Thesoft tissue mass extended from the distal $1 / 3$ of the humerus to the proximal $1 / 3$ of the radius. The physical characteristics and fast growing nature of the mass mandated further workup for possible 
malignancy. The preoperative MRI revealed a large soft tissue mass measuring $5.3 \times 5.7 \times 8.0 \mathrm{~cm}$ within the brachioradialis muscle that demonstrated lesional enhancement with the addition of contrast. These findings were consistent with a liposarcoma, which prompted a metastatic workup including a chest CT and incisional biopsy. The chest CT found no evidence of metastatic disease (Figure 1).

A thick yellow mass was identified during incisional biopsy. The specimen was sent for pathology. Histologically, the lesion was composed of bland spindle cells with short, stubby nuclei in a predominantly myxoidmatrix containing hyalinized collagen bundles, scattered mast cells, and a prominent adipocytic component. No atypia was seen.

The lesional cells were diffusely positive for CD34 and demonstrated focal desmin staining. Immuno histochemical stains for MDM2 and CDK4 were negative. An immunohistochemical stain for Rbwas negative, indicating a loss of expression of the $\mathrm{Rb}$ protein as a consequence of chromosome $13 q$ deletion. The differential diagnosis on initial evaluation included spindle cell lipoma, but the desmin positivity was more indicative of MMFB. Due to an unusual clinical presentation and MRI findings, the tissue was sent for a second opinion to the Department of Pathology at Brigham and Women's Hospital, Boston, MA, where the diagnosis of MMFB was confirmed (Figure 2).

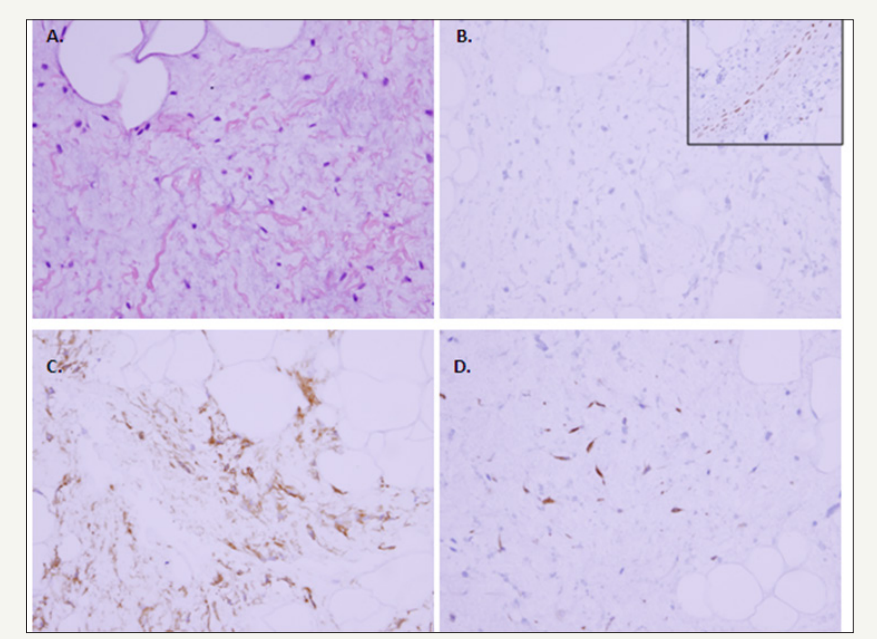

Figure 2: A. Hematoxylin and Eosin stain demonstrating short spindle cells, course collagen bundles and admixed fat in a myxoid stroma. B. Loss of expression of $\mathrm{Rb}$ protein (negative staining) with retained expression in non-neoplastic endothelial cells (brown staining, inset). C. Positive immunohistochemical staining for CD34 (brown staining). D. Focal positivity for desmin (brown staining).

Surgical excision of the mass was scheduled as definitive treatment. A $12 \mathrm{~cm}$ incision was madeutilizing an anterior approach overlying the left elbow joint. A plane between the brachioradialis and brachialis muscles was developed through which a large mass was apparent. It was densely adherent to the brachioradialis muscle. Through meticulous dissection, the mass was traced down to the joint, where there was no apparent capsular involvement. The mass was removed as a whole and sent to pathology with a confirmed diagnosis of MMFB.

The patient continues to do well and has had no recurrence or further complications at six month follow-up.

\section{Discussion}

In most cases benign masses of the upper extremity can be distinguished from malignant lesions based on their clinical presentation. Benign masses have a tendency to be soft, mobile, and slow growing, while malignant masses are more likely to be fast growing and immobile.Rare upper extremity tumors can fall outside of these general characteristics.

MMFB is a benign spindle cell tumor with neoplastic cells demonstrating variable fibromyofibroblastic differentiation [4,5]. MMFB fibroblasts characteristically demonstrate CD34 positivity, which is thoughtto indicategrowth from an uncommitted mesenchymal cell origin, capable of a wide array of differentiation. This may explain the many variants of MMFB, and the presence of fibroblasts, myofibroblasts, smooth muscle cells, adipocytes, chondrocytes and osseous cells within the same lesion [3]. Additionally, MMFB is characterized by positivity for vimentin, desmin and smooth muscle actin as well as loss of expression of $\mathrm{Rb}$ protein due to deletions of chromosome 13q [7].

Originally described as occurring in older males, MMFB has been found to have an equal distribution in postmenopausal women. Patients often report a history of slow growth over months to years [4]. Physical exam findings consist of a unilateral, solitary, firm and painless mass, frequently $1-4 \mathrm{~cm}$ in diameter [8]. MMFB has a predilection for areas associated with accessory breast tissue and other breast pathology such as the breast, axilla,abdominal wall and perineal region [2].

While MMFB is generally considered to present sporadically, there is documented association with gynecomastia and androgen deprivation therapy for prostate cancer [8,9]. Additionally, lesions characteristically stain for androgen, estrogen and progesterone receptors, which has resulted in speculation regarding thepossible pathogenic role of sex steroids $[2,4]$.

On MRI, MMFB presents as a well-circumscribed, nodular mass with homogeneous enhancement and internal septations that lack microcalcifications $[2,7]$. Successful diagnosis can often be made with the use of incisional biopsy. Gross examinationmay reveal a well circumscribed, smooth to lobulated rubbery mass with a solid pale white to grayish cut surface with a whorling pattern and a variable fatty component [2].

The differential diagnosis includes spindle cell lipoma and cellular angiofibroma, both of which show overlapping morphologic and immunophenotypic characteristics and demonstrate loss of chromosome 13q. Also in the differential is a variety of other bland looking spindle cell lesions such as nodular fasciitis, inflammatory 
pseudotumor, leiomyoma, angiomyolipoma,benign peripheral nerve sheath tumor and desmoid-type fibromatosis [7]. However, each has a unique immunohistochemical profile in reference to the presence or absence of desmin, smooth muscle actin, CD34, and Rbamong others $[6,10]$. Malignant tumors are typically ruled out based on the lack of nuclear pleomorphism, brisk mitotic activity, and curvilinear or plexiform vasculature [7].

MMFB is an uncommon benign stromal tumor, rarely found in the extremities. Making the diagnosis of MMFB can be difficult due to a radiological and clinical appearance that suggests more aggressive pathology. Our case is an example of the diagnostic challenge MMFB presents. Excision of MMFB is curative once the correct histological diagnosis is made.

\section{Statement of Human Rights}

All procedures followed were in accordance with the ethical standards of the responsible committee on human experimentation (institutional and national) and with the Helsinki Declaration of 1975, as revised in 2008 (5). Informed consent was obtained from all patients for being included in the study.

\section{Conflict of Interest}

The authors declare that they have no conflict of interest.

\section{Informed Consent}

Informed consent was obtained from all individual participants included in the study.

\section{Acknowledgements}

Gary S. Daum MD, Department of Pathology, Lankenau Medical Center

\section{References}

1. Wargotz ES, Weiss SW, Norris HJ (1987) Myofibroblastoma of the breast. sixteen cases of a distinctive benign mesenchymal tumor. Am J Surg Pathol 11(7): 493-502.

2. McMenamin ME, Fletcher CD (2001) Mammary-type myofibroblastoma of soft tissue: A tumor closely related to spindle cell lipoma. Am J Surg Pathol 25(8): 1022-1029.

3. Meguerditchian AN, Malik DA, Hicks DG, Kulkarni S (2008) Solitary fibrous tumor of the breast and mammary myofibroblastoma: The same lesion? Breast J 14(3): 287-292.

4. Magro G (2008) Mammary myofibroblastoma: A tumor with a wide morphologic spectrum. Arch Pathol Lab Med 132(11): 1813-1820.

5. Gocht A, Bosmuller HC, Bassler R, Tavassoli FA, Moinfar F et al. (1999) Breast tumors with myofibroblastic differentiation: Clinico-pathological observations in myofibroblastoma and myofibrosarcoma. Pathol Res Pract 195(1): 1-10.

6. Hamele-Bena D, Cranor ML, Sciotto C, Erlandson R, Rosen PP (1996) Uncommon presentation of mammary myofibroblastoma. Mod Pathol 9(7): 786-790.

7. Magro G, Salvatorelli L, Spadola S, Angelico G (2014) Mammary myofibroblastoma with extensive myxoedematous stromal changes: A potential diagnostic pitfall. Pathol Res Pract 210(12): 1106-1111.

8. Reis-Filho JS, Faoro LN, Gasparetto EL, Totsugui JT, Schmitt FC (2001) Mammary epithelioid myofibroblastoma arising in bilateral gynecomastia: Case report with immunohistochemical profile. Int J Surg Pathol 9(4): 331-334.

9. Morgan MB, Pitha JV (1998) Myofibroblastoma of the breast revisited: An etiologic association with androgens? Hum Pathol 29(4): 347-351.

10. Magro G, Michal M, Bisceglia M (2001) Benign spindle cell tumors of the mammary stroma: Diagnostic criteria, classification, and histogenesis. Pathol Res Pract 197(7): 453-466.

\section{Your subsequent submission with Crimson Publishers} will attain the below benefits

- High-level peer review and editorial services

- Freely accessible online immediately upon publication

- Authors retain the copyright to their work

- Licensing it under a Creative Commons license

- Visibility through different online platforms

- Global attainment for your research

- Article availability in different formats (Pdf, E-pub, Full Text)

- Endless customer service

- Reasonable Membership services

- Reprints availability upon request

- One step article tracking system 